\title{
The group logarithm past and present
}

\author{
T. Chinburg, G. Pappas and M. J. Taylor
}

October 3, 2011

\section{Introduction}

The group logarithm is a fundamental tool in the study of $K_{1}$ of the group ring $R[G]$ of a finite group $G$ and a $p$-adically complete ring of coefficients $R$. The goal of this article is to present both an accessible account of the basic theory of the group logarithm and also to give an overview of some of its most important applications.

Group logarithmic techniques were first used in the study of class groups of group rings, and in particular they were used to construct easily calculable homomorphic images of various subgroups of such class groups. In particular this produces completely new insights on class groups of group rings (see [T1,2,3]). There is a particularly readable account of this approach in Sect. 54 of [CR2]. R. Oliver used his own version of the group logarithm to study the subgroup $S K_{1}(R[G])$ of $p$-adic group rings when $R$ is the valuation ring of a finite extension of a $p$-adic field. There is a good account of the state of knowledge of such $S K_{1}$ groups prior to Oliver's work in Sect. 45 of [CR2]: from this one can see the major progress that his work represented.

The key application of the group logarithm which was the driver for the third named author's work, was the Fröhlich conjecture, which relates the Galois module structure of rings of algebraic integers to the constants occurring in the functional equations of certain Artin $L$-functions. The resolution of the Fröhlich conjecture involved two distinct uses of the group logarithm: the fixed point theorem for determinants (see Theorem 13 in Sect. 5), and the proof that certain Galois Gauss sums can be written as determinants of group ring elements (see Sect. 6.2).

In recent time the group logarithm has been extended to various Iwasawa algebras, with a view to tackling the non-commutative Iwasawa conjecture. (See 
for instance the initial work of Kato in $[\mathrm{K}]$ and then [Ka1-2] and [RW1].) This conjecture has now been proved (under various hypotheses) by J. Ritter and A. Weiss [RW2], and by M. Kakde [Ka3]. The main topic of this volume is to present a detailed account of Kakde's proof. The required extensions of the group logarithm are fully described elsewhere in this volume; see in particular the article of P. Schneider and O. Venjakob [SV].

A further recent use of the group logarithm has been in the construction of a second Chern class for suitable equivariant vector bundles on arithmetic surfaces, which can then be used to give an equivariant Riemann-Roch theorem (see [CPT3]). This requires the use of a group logarithm for group rings with higher dimensional coefficient rings. This development comes from [CPT1,2] and involves a substantial generalization of the original work of both Oliver and the third named author.

As explained above, it is our intention to provide a speedy and accessible introduction to the group logarithm, together with an overview of some of its many applications. For this reason we shall often focus on the case when $G$ is a $p$-group: this is in many ways the heart of the matter; one can then use induction techniques to obtain results for arbitrary finite groups. This article is therefore structured as follows. In Section 2 we define our notation and recall some standard results in $K$-theory. In Section 3 we describe the group logarithm for $p$-groups over a fairly general class of coefficient rings and in Section 4 we then use Oliver's work to describe $S K_{1}$ for such group rings. Then in Section 5 we give a very brief insight into the role of induction techniques: this Section is only a very cursory account: the reader is referred to [CPT1,2] and above all Ch. 12 in Oliver's book [O5] for more details. The article then concludes with an account of some of the most important applications of the group logarithm. We begin by describing some algebraic results including: the proof of Ullom's Conjecture on Swan groups and mentioning Oliver's description of class groups of group rings for $p$-groups. We then go on to consider two major applications of a more arithmetic flavor: the proof of the Fröhlich conjecture and the proof of an adelic Riemann-Roch theorem for an equivariant second Chern class. As indicated previously, a number of articles in this volume deal with applications to non-commutative Iwasawa theory, and so we avoid unnecessary duplication and shall not go into this key-application here. 


\section{Preliminaries}

\section{$2.1 \quad$ Notation.}

Throughout this article $R$ denotes an integral domain with field of fractions $N$ which has characteristic zero, and we write $N^{c}$ for a chosen algebraic closure of $N$. We impose two further conditions:

\section{Standing Hypotheses:}

(i) $R$ is $p$-adically complete, so that the natural map $R \rightarrow \varliminf_{n} R / p^{n} R$ is an isomorphism.

(ii) $R$ supports a lift of Frobenius; that is to say there is a ring endomorphism $F$ of $R$ with the property that $F(r) \equiv r^{p} \bmod p R$.

Examples of such rings $R$ are: the valuation ring of a non-ramified extension of the $p$-adic field $\mathbf{Q}_{p}$; the $p$-adic completion of the polynomial ring over $\mathbf{Z}_{p}$ in indeterminates $T_{1}, \ldots, T_{n}$

$$
\mathbf{Z}_{p}\left\langle\left\langle T_{1}, \ldots, T_{n}\right\rangle\right\rangle={\underset{\varliminf}{n}}_{\lim _{p}} \mathbf{Z}_{p}\left[T_{1}, \ldots, T_{n}\right] /\left(p^{n}\right) ;
$$

the $p$-adic completion of the ring of Laurent series over $\mathbf{Z}_{p}$ in indeterminates $T_{1}, \ldots, T_{n}$

$$
\mathbf{Z}_{p}\left\{\left\{T_{1}, \ldots, T_{n}\right\}\right\}={\underset{\varliminf}{n}}_{\lim _{n}} \mathbf{Z}_{p}\left(\left(T_{1}, \ldots, T_{n}\right)\right) /\left(p^{n}\right) .
$$

In each of the latter two examples we may take $F\left(T_{i}\right)=T_{i}^{p}$.

\subsection{Some elementary $K$-theory.}

Let $S$ denote a unitary ring (which is not necessarily commutative). In the usual way we define $G L(S)$ to be the direct limit of the groups $G L_{n}(S)$; in particular we view the group of units $S^{\times}=G L_{1}(S)$ as a subgroup of $G L(S)$ by embedding $S^{\times}$into $G L_{n}(S)$ as the diagonal matrices which are 1 in the non-leading diagonal positions. $E_{n}(S)$ denotes the group of $n \times n$ elementary matrices with coefficients in $S$, and we denote the direct limit of the $E_{n}(S)$ by $E(S)$. Then, in the usual way, the group $K_{1}(S)$ is defined as:

$$
K_{1}(S)=\frac{G L(S)}{E(S)} .
$$

For each $n>0$ we write $M_{n}(S)$ for the ring of $n \times n$ matrices with entries in $S$. If $S$ is commutative, then the determinant induces a homomorphism

$$
\operatorname{det}: K_{1}\left(M_{n}(S)\right) \rightarrow S^{\times}
$$


furthermore this map is an isomorphism if $S$ is also a local ring. See $[\mathrm{R}]$ for more details.

For a finite group $G$ we form the group ring $R[G]$. The group algebra $N^{c}[G]$ admits a natural algebra decomposition

$$
N^{c}[G]=\prod_{\chi} M_{n_{\chi}}\left(N^{c}\right)
$$

where the product extends over the irreducible $N^{c}$-characters of $G$. Hence, by the above, we know that we have

$$
K_{1}\left(N^{c}[G]\right)=\prod_{\chi}\left(N^{c}\right)^{\times} .
$$

We shall write Det for the composite map

$$
K_{1}(R[G]) \rightarrow K_{1}\left(N^{c}[G]\right)=\prod_{\chi}\left(N^{c}\right)^{\times} .
$$

The group $S K_{1}(R[G])$ is defined to be the kernel of Det, so that we have the exact sequence

$$
1 \rightarrow S K_{1}(R[G]) \rightarrow K_{1}(R[G]) \rightarrow \operatorname{Det}\left(K_{1}(R[G])\right) \rightarrow 1 .
$$

This exact sequence is fundamental for our understanding of $K_{1}(R[G])$ : note that $\operatorname{Det}\left(K_{1}(R[G])\right)$ and $S K_{1}(R[G])$ have rather different properties but the group logarithm gives great insight into both of these terms.

We shall write $\operatorname{Det}\left(R[G]^{\times}\right)$for the image of Det on the image of $R[G]^{\times}$in $K_{1}(R[G])$. In the case when $G$ is a $p$-group it follows easily by left and right multiplication by elementary matrices that:

Proposition $1 \operatorname{Det}\left(R[G]^{\times}\right)=\operatorname{Det}\left(K_{1}(R[G])\right)$.

Proof. See Theorem 1.2 in [CPT1].

If the group $G$ is abelian, then we have a diagram

$$
\begin{array}{ccc}
N^{c}[G]^{\times} & = & \prod_{\chi}\left(N^{c}\right)^{\times} \\
\uparrow & & \uparrow \\
R[G]^{\times} & \stackrel{\text { Det }}{\longrightarrow} & \operatorname{Det}\left(R[G]^{\times}\right)
\end{array}
$$

where the vertical maps are inclusions; hence, in this case, Det is injective on $R[G]^{\times}$and so we have shown:

Lemma 2 If $G$ is abelian, then $\operatorname{Det}: R[G]^{\times} \rightarrow \operatorname{Det}\left(R[G]^{\times}\right)$is an isomorphism. 


\section{The group logarithm.}

Throughout this section $G$ is a finite $p$-group. We write $I_{G}(R)$ for the augmentation ideal $\operatorname{ker}(\varepsilon: R[G] \rightarrow R)$, where the augmentation map $\varepsilon$ is the $R$-linear ring homomorphism with $\varepsilon(g)=1$ for all $g \in G$; and we let $J_{G}(R)$ denote the Jacobson radical of $R[G]$. When $R$ is clear from the context we shall write $I_{G}$ for $I_{G}(R)$ and $J_{G}$ for $J_{G}(R)$. Note that $I_{G}\left(\mathbf{F}_{p}[G]\right)$ is the Jacobson radical of the Artinian ring $\mathbf{F}_{p}[G]$ it therefore follows that we can find a positive integer $m$ such that $I_{G}\left(\mathbf{F}_{p}[G]\right)^{m}=0$. Note also that

$$
J_{G}(R) \equiv I_{G}(R)=R I_{G}\left(\mathbf{Z}_{p}\right) \bmod p R[G]
$$

and so

$$
I_{G}(R)^{m} \subset p R[G], \quad J_{G}(R)^{m} \subset p R[G] .
$$

Therefore, for $x \in J_{G}(R)$, the logarithmic series

$$
\log (1-x)=-\sum_{n>0} \frac{x^{n}}{n}
$$

converges to an element of $N[G]$. We now use passage to conjugacy classes, together with a $p$ th power map, to produce a logarithm which converges to integral values. Let $\operatorname{Conj}(G)$ denote the set of conjugacy classes of $G$ and let $\tau=\tau_{G}: N[G] \rightarrow N[\operatorname{Conj}(G)]$ be the $N$-linear map which maps each group element to its conjugacy class. We define the $p$-th power operation $\Psi=\Psi_{F}$ to be the $F$-semi-linear endomorphism of $R[\operatorname{Conj}(G)]$ which maps the conjugacy class of each group element to the conjugacy class of its $p$ th power; so that for $r \in R, g \in G$

$$
\Psi(r \tau(g))=F(r) \tau\left(g^{p}\right) .
$$

We then define

$$
\begin{aligned}
L(1-x) & =-\sum_{n>0} \frac{\tau\left(x^{n}\right)}{n}+\sum_{n>0} \frac{\Psi \circ \tau\left(x^{n}\right)}{n p} \\
& =-\sum_{p \nmid n>0} \frac{\tau\left(x^{n}\right)}{n}-\sum_{n>0} \frac{\tau\left(x^{n p}\right)}{n p}+\sum_{n>0} \frac{\Psi \circ \tau\left(x^{n}\right)}{n p} \\
& =-\sum_{p \nmid n>0} \frac{\tau\left(x^{n}\right)}{n}+\sum_{n>0} \frac{\Psi \circ \tau\left(x^{n}\right)-\tau\left(x^{n p}\right)}{n p} .
\end{aligned}
$$

Theorem 3

$$
L\left(1+J_{G}(R)\right) \subset R[\operatorname{Conj}(G)] \text { and } L\left(1+I_{G}(R)\right) \subset \tau\left(I_{G}(R)\right) .
$$

Although the group logarithm is defined on all of $1+J_{G}(R)$, we shall often restrict it to $1+I_{G}(R)$ as this often gives a cleaner statement of results (see for example Theorem 8). 
The proof of the theorem comes from a straightforward non-commutative version of the binomial theorem (see 6.2 in [T1] and Lemma 5.3 in Ch. 2 in [F1] for details). We need to prove that for each $n>0$

$$
\Psi \circ \tau\left(x^{n}\right)-\tau\left(x^{n p}\right) \in n p R[\operatorname{Conj}(G)] .
$$

The key-idea can be seen from the case when the group $G$ is abelian: indeed, in that case $\tau$ is the identity and we know by the usual binomial theorem that for any $x=\sum r_{g} g \in R[G]$

$$
x^{p}=\left(\sum r_{g} g\right)^{p} \equiv \sum r_{g}^{p} g^{p} \equiv \sum F\left(r_{g}\right) g^{p}=\Psi(x) \bmod p R[G]
$$

and from this it follows easily that for any $n>0$

$$
x^{n p} \equiv \Psi(x)^{n}=\Psi\left(x^{n}\right) \bmod n p R[G] .
$$

We relate the logarithm to Det via the following lemma which depends on the well known fact that "the logarithm of the determinant is trace of the logarithm":

Lemma 4 Let $\chi$ be an $N^{c}$-valued virtual character of $G$. Recall that the $p$-th Adams operation on $\chi$ is defined by the formula $\psi^{p} \chi(g)=\chi\left(g^{p}\right)$ and $\psi^{p} \chi$ is also a virtual character of $G$. We extend $F$ to $R[G]$ by coefficientwise action; that is to say

$$
F\left(\sum r_{g} g\right)=\sum F\left(r_{g}\right) g
$$

Then, for any $x \in I_{G}(R)$ we have

$$
p \chi(L(1-x))=\log \left(\operatorname{Det}_{p \chi}(1-x)\right)-\log \left(\operatorname{Det}_{\psi^{p}} \chi(1-F(x))\right) .
$$

$L$ is a homomorphism since the characters of $G$ span the dual of $N[\operatorname{Conj}(G)]$. Since $\operatorname{Det}_{\chi}(1-x)=1$ implies that $\operatorname{Det}_{\chi}(1-F(x))=1$, it follows from (9) that $\operatorname{ker}($ Det $) \subset \operatorname{ker}(L)$, and so there is a unique map $\nu: \operatorname{Det}\left(1+I_{G}\right) \rightarrow \tau\left(I_{G}\right)$ which makes the following diagram commutative:

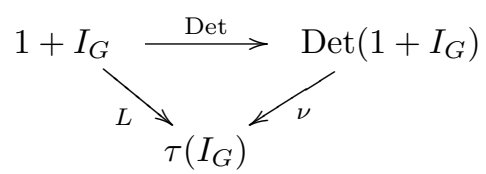

As $\tau\left(I_{G}\right) \subset N[\operatorname{Conj}(G)]$, clearly the elements of $\operatorname{Det}\left(1+I_{G}\right)$ of finite order lie in $\operatorname{ker} \nu$. Using the fact that $\psi^{p}$ is nilpotent on virtual characters of degree zero, it easy to establish the reverse inclusion: namely, that $\operatorname{ker} \nu$ is contained in the subgroup elements of $\operatorname{Det}\left(1+I_{G}\right)$ of finite order. A result of C.T.C Wall ([W1,2]) extends readily (see Theorems 3.14 and 3.15 in [CPT1]) to give: 


\section{Theorem 5}

$$
\operatorname{ker} \nu=\operatorname{Det}(G) \text {. }
$$

In Lemma 2 we have seen that Det is an isomorphism on $R\left[G^{\mathrm{ab}}\right]^{\times}$; to get a complete picture of Det on all of $R[G]$ we now consider the ideal:

$$
A_{G}(R)=\operatorname{ker}\left(R[G] \rightarrow R\left[G^{\mathrm{ab}}\right]\right) .
$$

Wall's theorem tells us immediately that $\nu$ is injective on $\operatorname{Det}\left(1+A_{G}\right)$. The fundamental result in the theory of the group logarithm is the fact that the image of $\nu$ on $\operatorname{Det}\left(1+A_{G}\right)$ is a natural $R$-lattice (see Theorem 3.5 in [CPT1]):

Theorem 6 We have the equality in $N[\operatorname{Conj}(G)]$

$$
\nu\left(\operatorname{Det}\left(1+A_{G}\right)\right)=\tau\left(A_{G}\right) .
$$

We can then piece this result together the abelian result to get the important exact sequences:

$$
\begin{aligned}
& 1 \rightarrow \tau\left(A_{G}\right) \rightarrow \operatorname{Det}\left(R[G]^{\times}\right) \rightarrow R\left[G^{\mathrm{ab}}\right]^{\times} \rightarrow 1 \\
& 1 \rightarrow \tau\left(A_{G}\right) \rightarrow \operatorname{Det}\left(1+I_{G}(R)\right) \rightarrow 1+I_{G^{\mathrm{ab}}}(R) \rightarrow 1 .
\end{aligned}
$$

Application. As a first illustration of the power of the group logarithm, we use this exact sequence to prove a fixed point theorem for determinants for group rings over $p$-groups. Let $S$ be a finite Galois extension of $R$ with $\Delta=\operatorname{Gal}(S / R)$, and suppose that $S$ also satisfies the Standing Hypotheses in Section 2. For the sake of simplicity we shall assume that the lift of Frobenius $F_{S}$ of $S$ is compatible with the lift of Frobenius $F_{R}$ of $R$ in the sense that $\left.F_{S}\right|_{R}=F_{R}$. We let $\Delta$ act on $\operatorname{Det}\left(S[G]^{\times}\right)$via coefficients; so that for $\delta \in \Delta, x \in S[G]^{\times}$we have $\operatorname{Det}(x)^{\delta} \stackrel{\text { defn }}{=} \operatorname{Det}\left(x^{\delta}\right)$. Clearly $\operatorname{Det}\left(R[G]^{\times}\right) \subset \operatorname{Det}\left(S[G]^{\times}\right)^{\Delta}$. Using the group logarithm we can now show:

\section{Theorem 7}

$$
\operatorname{Det}\left(R[G]^{\times}\right)=\operatorname{Det}\left(S[G]^{\times}\right)^{\Delta} .
$$

Remark. In fact, with a bit more effort, we can avoid the condition that the lifts of Frobenius are compatible; see Section 4 of [CPT1] for details.

Proof. First note that

$$
\tau\left(A_{G}(S)\right)=S \otimes_{R} \tau\left(A_{G}(R)\right), \quad\left(S\left[G^{\mathrm{ab}}\right]^{\times}\right)^{\Delta}=R\left[G^{\mathrm{ab}}\right]^{\times} .
$$


By the naturality of the group logarithm (which depends on a compatible choice of lifts of Frobenius, so that $\left.L_{S[G]}\right|_{R[G]}=L_{S[G]}$ ), from (11) we have the commutative diagram:

$$
\begin{aligned}
& 1 \rightarrow \tau\left(A_{G}(R)\right) \quad \rightarrow \quad \operatorname{Det}\left(R[G]^{\times}\right) \quad \rightarrow \quad R\left[G^{a b}\right]^{\times} \quad \rightarrow \quad 1 \\
& \downarrow \quad \downarrow \quad \downarrow= \\
& 1 \rightarrow\left(S \otimes_{R} \tau\left(A_{G}(R)\right)\right)^{\Delta} \rightarrow \operatorname{Det}\left(S[G]^{\times}\right)^{\Delta} \rightarrow\left(S\left[G^{\mathrm{ab}}\right]^{\times}\right)^{\Delta} \rightarrow 1
\end{aligned}
$$

with the bottom row exact since $S \otimes_{R} \tau\left(A_{G}(R)\right)$ is $R[\Delta]$-projective, since $S$ is $R[\Delta]$-projective.

Since the left and right vertical arrows are equalities the central vertical arrow is also an equality.

We now complete our understanding of the group logarithm by describing the cokernel of the map $\nu: \operatorname{Det}\left(1+I_{G}\right) \rightarrow \tau\left(I_{G}\right)$. The evaluation of this cokernel was first carried out by R. Oliver in [O 2] for the case when $R$ is a non-ramified $p$-adic ring of integers:

Theorem 8 Let $i_{G}$ denote the inclusion $\nu\left(\operatorname{Det}\left(1+I_{G}\right)\right) \hookrightarrow \tau\left(I_{G}\right)$; then there is a natural isomorphism:

$$
\operatorname{coker}\left(i_{G}\right) \cong G^{\mathrm{ab}} \otimes_{\mathbf{z}} \frac{R}{(1-F) R} .
$$

Sketch proof. (For a full proof see 3.d in [CPT1].) First we observe that by Theorem $6, \operatorname{coker}\left(i_{G}\right) \cong \operatorname{coker}\left(i_{G^{\mathrm{ab}}}\right)$ and so without loss of generality we may suppose $G$ to be abelian. We then have the standard differential map for augmented commutative rings:

$$
d: R[G] \rightarrow \Omega_{R[G] / R}^{1}=\frac{I_{G}(R)}{I_{G}(R)^{2}}=G \otimes_{\mathbf{z}} R, \quad x \longmapsto x-\varepsilon(x) \bmod I_{G}(R)^{2} .
$$

Thus $F$ acts naturally on $\Omega_{R[G] / R}^{1}$ via $R$. Recall that we also have the $R$ semilinear endomorphism $\Psi$ of $R G$ ] with the property that for $r \in R, g \in$ $G, \Psi(r g)=F(r) g^{p}$; and moreover, since $G$ is now abelian, $\Psi$ is now a ring homomorphism. By using lifts to characteristic zero, it is possible to give a sense to the following equality for each $x \in I_{G}$

$$
F(d x)=\frac{1}{p} d \Psi(x)
$$

see 3.d in [CPT1] for details. Then we have the equalities:

$$
\begin{aligned}
d(L(1-x)) & =d(\log (1-x))-d\left(\log \frac{1}{p} \Psi(1-x)\right) \\
& =-d x+F d x=(F-1) d x
\end{aligned}
$$


and so $d\left(\nu\left(1+I_{G}\right)\right) \subset(1-F)(R \otimes G)=(1-F) R \otimes G$. It is then relatively straightforward to show the equality $d\left(\nu\left(1+I_{G}\right)\right)=(1-F) R \otimes G$ and to see that $d$ induces an isomorphism

$$
\frac{1+I_{G}}{\nu\left(1+I_{G}\right)} \cong \frac{R}{(F-1) R} \otimes G
$$

\section{$4 \quad S K_{1}(R[G])$}

In this section we suppose that $G$ is a $p$-group and that, in addition to the Standing Hypotheses, $p R$ is now a prime ideal of $R$; although it is not essential, we shall also suppose, for the sake of presentational simplicity, that $S K_{1}(R)=$ $\{1\}$. For the treatment of the more general case when $S K_{1}(R)$ is non-trivial see [CPT2]. We now follow R. Oliver and use the more general group logarithm to describe $S K_{1}(R[G])$.

Recall that in general we have the long exact sequence of $K$-theory

$$
\cdots \rightarrow K_{2}(R) \rightarrow K_{1}\left(R[G], I_{G}\right) \rightarrow K_{1}(R[G]) \rightarrow K_{1}(R) \rightarrow \cdots
$$

We write $K_{1}^{\prime}\left(R[G], I_{G}\right)$ for the image of $K_{1}\left(R[G], I_{G}\right)$ in $K_{1}(R[G])$. We denote the Whitehead group $K_{1}^{\prime}\left(R[G], I_{G}\right) / \operatorname{Im}(G)$ by $\mathrm{Wh}_{G}^{\prime}(R)$, or $\mathrm{Wh}_{G}^{\prime}$ when $R$ is clear from the context. Since $S K_{1}(R)=\{1\}$ and since $S K_{1}(R[G]) \cap[G]=\{1\}$, we note that $S K_{1}(R[G])$ is naturally a subgroup of $\operatorname{Wh}_{G}^{\prime}(R)$. Using Theorems 5 and 8 we get the exact sequence:

$$
1 \rightarrow \frac{\operatorname{Det}\left(1+I_{G}\right)}{\operatorname{Det}(G)} \rightarrow \tau\left(I_{G}\right) \rightarrow G^{\mathrm{ab}} \otimes \mathbf{z} \frac{R}{(1-F) R} \rightarrow 1
$$

and using the exact sequence (5), together with our hypothesis that $S K_{1}(R)=$ $\{1\}$, we get the further exact sequence

$$
1 \rightarrow S K_{1}(R[G]) \rightarrow \mathrm{Wh}_{G}^{\prime}(R) \rightarrow \frac{\operatorname{Det}\left(1+I_{G}\right)}{\operatorname{Det}(G)} \rightarrow 1 .
$$

These two exact sequences may be spliced to together to give the four term exact sequence

$$
1 \rightarrow S K_{1}(R[G]) \rightarrow \mathrm{Wh}_{G}^{\prime}(R) \rightarrow \tau\left(I_{G}\right) \rightarrow G^{\mathrm{ab}} \otimes \mathbf{z} \frac{R}{(1-F) R} \rightarrow 1 .
$$

Suppose we have an extension of finite $p$-groups

$$
1 \rightarrow H \stackrel{i}{\rightarrow} \widetilde{G} \stackrel{\alpha}{\rightarrow} G \rightarrow 1
$$


then we have a commutative diagram:

$$
\begin{aligned}
& 1 \rightarrow S K_{1}(R[H]) \rightarrow \mathrm{Wh}_{H}^{\prime} \rightarrow \tau_{H}\left(I_{H}\right) \stackrel{\leftrightarrow--}{\rightarrow} H^{\mathrm{ab}} \otimes \mathbf{z} \frac{R}{(1-F) R} \rightarrow 1 \\
& \downarrow i_{*} \quad \downarrow \quad \downarrow \quad \downarrow i^{a b} \otimes 1 \\
& 1 \rightarrow S K_{1}(R[\widetilde{G}]) \rightarrow \mathrm{Wh}_{\widetilde{G}}^{\prime} \stackrel{\leftarrow--}{\rightarrow} \tau_{\widetilde{G}}\left(I_{\widetilde{G}}\right) \quad \rightarrow \quad \widetilde{G}^{\mathrm{ab}} \otimes \mathbf{z} \frac{R}{(1-F) R} \rightarrow 1 \\
& \downarrow \alpha_{*} \quad \downarrow \quad \downarrow \quad \downarrow \alpha^{a b} \otimes 1 \\
& 1 \rightarrow S K_{1}(R[G]) \rightarrow \mathrm{Wh}_{G}^{\prime} \rightarrow \tau_{G}\left(I_{G}\right) \quad \rightarrow \quad G^{\mathrm{ab}} \otimes \mathbf{z} \frac{R}{(1-F) R} \rightarrow 1 .
\end{aligned}
$$

We then use the the dotted arrows, which are just set-theoretic lifts, - in a similar manner to the construction to the boundary map in the snake lemma, but done twice - to produce a map:

$$
\Delta: \operatorname{ker}\left(i_{G}^{\mathrm{ab}}\right) \otimes \mathbf{z} \frac{R}{(1-F) R} \rightarrow \operatorname{coker}\left(\alpha_{*}\right) .
$$

Next we consider the following two subgroups of $G$ :

$$
\begin{aligned}
& H_{0}=H \cap[\widetilde{G}, \widetilde{G}] \\
& \left.H_{1}=\langle h \in H| h=\left[\widetilde{g}_{1}, \widetilde{g}_{2}\right] \text { for } \widetilde{g}_{1}, \widetilde{g}_{2} \in \widetilde{G}\right\rangle ;
\end{aligned}
$$

that is to say $H_{1}$ is generated by $\widetilde{G}$ commutators which lie in $H$, so that obviously $[H, H] \subset H_{1} \subset H_{0}$ and $\operatorname{ker}\left(i_{G}^{\mathrm{ab}}\right)=H_{0} /[H, H]$. We write $\widetilde{\kappa}_{\alpha}$ for the composite map

$$
\widetilde{\kappa}_{\alpha}: H_{0} \otimes \mathbf{z} \frac{R}{(1-F) R} \rightarrow \operatorname{ker}\left(i_{G}^{a b}\right) \otimes \mathbf{z} \frac{R}{(1-F) R} .
$$

As in Proposition 16 in [O2] (see also 3.3 in [CPT2]) we know that

Proposition 9 The map $\widetilde{\kappa}_{\alpha}$ induces an isomorphism

$$
\kappa_{a}: \frac{H_{0}}{H_{1}} \otimes \mathbf{z} \frac{R}{(1-F) R} \rightarrow \operatorname{coker}\left(\alpha_{*}\right) .
$$

Suppose now that the extension (15) is a central extension. From [O2] and [CPT2] we have:

Theorem 10 (a) (Hopf) There is a natural map $\delta_{\alpha}^{*}: \mathrm{H}_{2}(G, \mathbf{Z}) \rightarrow H$ and $\operatorname{Im}\left(\delta_{\alpha}^{*}\right)=H_{0}$;

(b) Define $\mathrm{H}_{2}^{\mathrm{ab}}(G, \mathbf{Z})$ to be the subgroup of $\mathrm{H}_{2}(G, \mathbf{Z})$ generated by the images of the $\mathrm{H}_{2}(A, \mathbf{Z})$ for all abelian subgroups of $G$; then $\delta_{\alpha}^{*}\left(\mathrm{H}_{2}^{\mathrm{ab}}(G, \mathbf{Z})\right)=H_{1}$.

We define

$$
\overline{\mathrm{H}}_{2}(G, \mathbf{Z})=\frac{\mathrm{H}_{2}(G, \mathbf{Z})}{\mathrm{H}_{2}^{\mathrm{ab}}(G, \mathbf{Z})}
$$


Piecing the above together we get maps

$$
S K_{1}(R[G]) \rightarrow \operatorname{coker}\left(\alpha_{*}\right) \stackrel{\kappa_{\alpha}^{-1}}{\cong} \frac{H_{0}}{H_{1}} \otimes \mathbf{z} \frac{R}{(1-F) R} \stackrel{\delta_{\alpha}^{*}}{\longleftarrow} \overline{\mathrm{H}}_{2}(G, \mathbf{Z}) \otimes \mathbf{z} \frac{R}{(1-F) R} .
$$

By considering families of central extensions, as above, Oliver shows that it is possible to find situations where $\delta_{\alpha}^{*}$ is injective (and hence an an isomorphism); this then yields a surjective map (which by naturality is independent of the particular such central extension and map $\alpha$ used)

$$
\Theta: S K_{1}(R[G]) \rightarrow \overline{\mathrm{H}}_{2}(G, \mathbf{Z}) \otimes \mathbf{z} \frac{R}{(1-F) R}
$$

The following result is due to Oliver for $p$-adic rings of integers; it was generalized to rings of integers of the completed maximal non-ramified extension of $\mathbf{Q}_{p}$ in [IV]; and in [CPT2] it is proved for general $R$ in which the ideal $p R$ is prime and which satisfy the Standing Hypotheses of this article.

Theorem 11 The map $\Theta$ is an isomorphism which is natural in $G$ and $R$.

\section{Induction methods.}

We have seen that the group logarithm is a powerful tool for the study of $K_{1}(R[G])$ when $G$ is a $p$-group. There are a number of induction techniques which allow us to use results for $p$-groups to obtain results for arbitrary finite groups. In this section we assume that, in addition to satisfying the Standing Hypotheses, $R$ is also a Noetherian and normal ring. Then by Theorem 1.2 in [CPT1] we have:

Theorem 12 For an arbitrary finite group $G$ and for $R$ as above

$$
\operatorname{Det}\left(K_{1}(R[G])\right)=\operatorname{Det}\left(R[G]^{\times}\right)
$$

In Chapter 11 of [O5], Oliver presents an extremely effective set of induction techniques using Green rings and Mackey functors - building on work of Dress in [D]. For instance, under suitable circumstances, this allows one to describe a result for an arbitrary finite group $G$ as a direct limit over the elementary $p$-groups of $G$. In [Sn], V. Snaith exhibits a technique of explicit Brauer induction. This has the remarkable property of being natural with respect to Adams operations. Since the $p$ th Adams operation plays a key-role in the properties the group logarithm (see for instance Lemma 4), Snaith's method is particularly well adapted to inductive techniques involving the group logarithm. In a number of situations, however, it suffices to use the simpler technique of character 
action due to S. Ullom. We briefly illustrate this method by sketching a proof of the following general version of the fixed point Theorem 7 :

Theorem 13 Let $G$ be an arbitrary finite group and we keep the notation of Theorem 7. Suppose that, in addition to the Standing Hypotheses, $S$ is both Noetherian and normal. Then

$$
\operatorname{Det}\left(K_{1}(S[G])\right)^{\Delta}=\operatorname{Det}\left(K_{1}(R[G])\right) .
$$

\subsection{Character action on $K_{1}$}

We let $G_{0}\left(\mathbf{Z}_{p}[G]\right)$ denote the Grothendieck group of finitely generated $\mathbf{Z}_{p}[G]$ modules and let $G_{0}^{\mathbf{Z}_{p}}\left(\mathbf{Z}_{p}[G]\right)$ denote the Grothendieck group of finitely generated $\mathbf{Z}_{p}[G]$-modules which are projective over $\mathbf{Z}_{p}$. From 38.42 and 39.9 in [CR2] we have:

\section{Proposition 14}

$$
G_{0}^{\mathbf{Z}_{p}}\left(\mathbf{Z}_{p}[G]\right) \cong G_{0}\left(\mathbf{Z}_{p}[G]\right) \cong G_{0}\left(\mathbf{Q}_{p}[G]\right)
$$

with the first isomorphism induced by the forgetful map and the second isomorphism induced by the extension of scalars map $\otimes_{\mathbf{z}_{p}} \mathbf{Q}_{p}$.

Proposition 15 Let $R$ be an integral domain containing $\mathbf{Z}_{p}$. Then $G_{0}^{\mathbf{Z}_{p}}\left(\mathbf{Z}_{p}[G]\right)$ and hence, by the previous proposition, $G_{0}\left(\mathbf{Q}_{p}[G]\right)$, acts naturally on $K_{1}(R[G])$ via the rule that for an $\mathbf{Z}_{p}[G]$-lattice $L$ and for an element of $K_{1}(R[G])$ represented by a pair $(P, \alpha)$ (where $P$ is a projective $R[G]$-module and $\alpha$ is an $R[G]$-automorphism of $P)$, then

$$
L \cdot(P, \alpha)=\left(\left(L \otimes \mathbf{z}_{p} P\right),\left(1 \otimes \mathbf{z}_{p} \alpha\right)\right) .
$$

The functor $G \mapsto K_{1}(R[G])$ is a Frobenius module for the Frobenius functor $G \mapsto G_{0}^{\mathbf{Z}_{p}}\left(\mathbf{Z}_{p}[G]\right)$ (see page 4 in [CR2] and also $\left.[L]\right)$. $S K_{1}(R[G])$ is a Frobenius submodule of $K_{1}(R[G])$ and therefore the action of $G_{0}\left(\mathbf{Q}_{p}[G]\right)$ on $K_{1}(R[G])$ induces an action on $\operatorname{Det}(G L(R[G])$ ). (See Ullom's Theorem in 2.1 of [T1], and see also below for his explicit description of this action).

Proof. From Ex 39.5 in [CR2] we know that $G \mapsto K_{1}\left(\mathbf{Z}_{p}[G]\right)$ is a Frobenius module for $G_{0}^{\mathbf{Z}_{p}}$. Moreover, the extension of scalars map $G_{0}^{\mathbf{Z}_{p}}\left(\mathbf{Z}_{p}[G]\right) \rightarrow$ $G_{0}^{R}(R[G])$ is a morphism of Frobenius modules over $G_{0}^{\mathbf{Z}_{p}}\left(\mathbf{Z}_{p}[G]\right)$ by 38.11 loc. cit.. This then establishes the first part of the proposition. Let $N$ denote the field of fractions of $R$. Because $G_{0}^{R}(R[G]) \rightarrow G_{0}\left(N^{c}[G]\right)$ is similarly a morphism of Frobenius modules over $G_{0}^{R}(R[G])$, it follows that the kernel

$$
S K_{1}(R[G])=\operatorname{ker}\left(K_{1}(R[G]) \rightarrow K_{1}\left(N^{c}[G]\right)\right)
$$


is also a Frobenius module over $G_{0}^{R}(R[G])$.

Next we recall Ullom's explicit formula for the action of the character ring $G_{0}\left(\mathbf{Q}_{p}[G]\right)$ on $\operatorname{Det}(G L(R[G]))$.

We view $G_{0}\left(\mathbf{Q}_{p}[G]\right)$ as the ring of virtual characters of finitely generated $\mathbf{Q}_{p}[G]$-modules and we let $\phi \in K_{0}\left(\mathbf{Q}_{p}^{c}[G]\right.$ ) (which we identify with the ring of virtual $\mathbf{Q}_{p}^{c}$-valued characters of $\left.G\right)$, and let $r \in G L(R[G])$ correspond to the pair $(P, \alpha)$ under the two descriptions of $K_{1}(R[G])$. Then Ullom has shown that the induced Frobenius action of $G_{0}\left(\mathbf{Q}_{p}[G]\right)$ is given explicitly by

$$
\theta \cdot(P, \alpha)=\theta \cdot(\phi \mapsto \operatorname{Det}(r)(\phi))=(\phi \mapsto \operatorname{Det}(r)(\bar{\theta} \phi))
$$

where $\bar{\theta}$ denotes the contragredient of the character $\theta$. In particular we have

$$
\left(\operatorname{Ind}_{H}^{G} \theta\right) \cdot \operatorname{Det}(r)=\operatorname{Ind}_{H}^{G}\left(\theta \cdot\left(\operatorname{Res}_{G}^{H}(\operatorname{Det}(r))\right)\right)
$$

which is one of the standard identities for Frobenius modules. The proofs of these standard facts are exactly the same as the proofs in Chapter 2 pages 21-25 in [T1].

\subsection{Brauer Induction}

For a given positive integer $m, \mu_{m}$ denotes the group of roots of of unity of order $m$ in $\mathbf{Q}_{p}^{c}$. We then identify $\operatorname{Gal}\left(\mathbf{Q}_{p}\left(\mu_{m}\right) / \mathbf{Q}_{p}\right)$ as a subgroup of $(\mathbf{Z} / m \mathbf{Z})^{\times}$ in the usual way. Let $l$ be a prime number. Recall that a semi-direct product of a cyclic group $C$ (of order $m$, say, which is coprime to $l$ ) by an $l$-group $L$, $C \rtimes L$, is called $\mathbf{Q}_{p}$-l-elementary (see page 112 in [S]) if for given $\lambda \in L$ there exists

$$
t=t(\lambda) \in \operatorname{Gal}\left(\mathbf{Q}_{p}\left(\mu_{m}\right) / \mathbf{Q}_{p}\right) \subseteq(\mathbf{Z} / m \mathbf{Z})^{\times}
$$

such that for all $c \in C$

$$
\lambda c \lambda^{-1}=c^{t} .
$$

Theorem 16 For a given finite group $G$, there exists an integer $m$ coprime to $l$ such that

$$
m G_{0}\left(\mathbf{Q}_{p}[G]\right) \subseteq \sum_{J} \operatorname{Ind}_{J}^{G}\left(G_{0}\left(\mathbf{Q}_{p}[J]\right)\right)
$$

where $J$ ranges over the $\mathbf{Q}_{p}$-l-elementary subgroups of $G$.

Proof. See Theorem 28 in [S]. 


\section{$5.3 \mathrm{Q}_{p}$-p-elementary groups}

Suppose now that $G$ is a $\mathbf{Q}_{p}$-p-elementary group, so that $G$ may be written as a semi-direct product $C \rtimes P$, where $C$ is a cyclic normal subgroup of order $s$, which is prime to $p$, and where $P$ is $p$-group. We decompose the commutative group ring $\mathbf{Z}_{p}[C]$ according as the divisors $m$ of $s$

$$
\mathbf{Z}_{p}[C]=\prod_{m} \mathbf{Z}_{p}[m],
$$

where $\mathbf{Z}_{p}[m]$ is the semi-local ring

$$
\mathbf{Z}_{p}[m]=\mathbf{Z}\left[\zeta_{m}\right] \otimes \mathbf{z} \mathbf{Z}_{p}
$$

and where $\zeta_{m}$ is a primitive $m$ th root of unity. We set $R_{m}=R \otimes \mathbf{z}_{p} \mathbf{Z}_{p}[m]$, although $R_{m}$ is not an integral domain, by Section 6 in [CPT1] we know that $R_{m}$ decomposes as a product of integral domains each of which satisfies the Standing Hypotheses.

For each $m$ the conjugation action of $P$ on $C$ induces a homomorphism $\alpha_{m}: P \rightarrow \operatorname{Aut}\left\langle\zeta_{m}\right\rangle$ and we let $H_{m}=\operatorname{ker}\left(\alpha_{m}\right)$ and $A_{m}=\operatorname{Im}\left(\alpha_{m}\right)$. When $m$ has been fixed we shall feel free to drop the index $m$.

Tensoring the decomposition (18) with $\otimes_{\mathbf{z}_{p}[C]} R[G]$ affords a decomposition of $R$-algebras

$$
R[G]=\prod_{m} R[m] \circ P
$$

where $R[G] \circ P$ denotes the natural twisted group ring. We shall study the group $\operatorname{Det}\left(R[G]^{\times}\right)$by studying the various subgroups $\operatorname{Det}\left(R[m] \circ P^{\times}\right)$. Note that the twisted group ring $R[m] \circ P$ contains the standard group ring $R[m]\left[H_{m}\right]$. We therefore have the inclusion map $i: R[m]\left[H_{m}\right] \rightarrow R[m] \circ P$. We also have a restriction map defined by choosing a transversal $\left\{a_{i}\right\}$ of $P / H_{m}$, which induces a restriction homomorphism res $\left.: R[m] \circ P^{\times} \rightarrow G L_{|A|}\left(R[m]\left[H_{m}\right]\right]\right)$ and hence using Theorem 12 above:

$$
r_{m}: \operatorname{Det}\left(R[m] \circ P^{\times}\right) \rightarrow \operatorname{Det}\left(G L_{|A|}\left(R[m]\left[H_{m}\right]\right)\right) \cong \operatorname{Det}\left(R[m]\left[H_{m}\right]^{\times}\right) .
$$

Since for $\pi \in P, x \in R[m] \circ P^{\times}$, we know that $\operatorname{Det}\left(\pi x \pi^{-1}\right)=\operatorname{Det}(x)$, we see that

$$
r_{m}: \operatorname{Det}\left(R[m] \circ P^{\times}\right) \rightarrow\left(\operatorname{Det}\left(R[m]\left[H_{m}\right]^{\times}\right)\right)^{A_{m}} .
$$

Here $A_{m}$ acts via $\alpha_{m}$ on $R[m]$ and by conjugation on $H_{m}$. One can then relatively easily show (see Theorem 6.2 in [CPT1]):

Theorem 17 The map $r_{m}$ gives an isomorphism between $\operatorname{Det}\left(R[m] \circ P^{\times}\right)$and $\operatorname{Det}\left(R[m]\left[H_{m}\right]^{\times}\right)^{A_{m}}$. 
Finally we show:

Theorem 18 Let $S$ and $R$ be as stated in Theorem 13 and let $G$ be a finite $\mathbf{Q}_{p}$-p-elementary group, then

$$
\operatorname{Det}\left(S[G]^{\times}\right)^{\Delta}=\operatorname{Det}\left(R[G]^{\times}\right) .
$$

Proof. By (19) and Theorem 17 above

$$
\begin{aligned}
\operatorname{Det}\left(S[G]^{\times}\right)^{\Delta} & =\oplus_{m} \operatorname{Det}\left(S[m] \circ P^{\times}\right)^{\Delta} \\
& =\oplus_{m}\left(\operatorname{Det}\left(S[m]\left[H_{m}\right]^{\times}\right)^{A_{m}}\right)^{\Delta} .
\end{aligned}
$$

As the actions of $\Delta$ and $A_{m}$ commute on $S[m]\left[H_{m}\right]=S \otimes_{R} R[m]\left[H_{m}\right]$ ( $\Delta$ acts via the first term and $A_{m}$ acts via the second term), we see that

$$
\operatorname{Det}\left(S[G]^{\times}\right)^{\Delta}=\oplus_{m}\left(\operatorname{Det}\left(S[m]\left[H_{m}\right]^{\times}\right)^{\Delta}\right)^{A_{m}}
$$

and so by Theorem 7 we have equalities

$$
\begin{aligned}
\operatorname{Det}\left(S[G]^{\times}\right)^{\Delta} & =\oplus_{m} \operatorname{Det}\left(R[m]\left[H_{m}\right]^{\times}\right)^{A_{m}} \\
& =\oplus_{m} \operatorname{Det}\left(R[m] \circ P^{\times}\right) \\
& =\operatorname{Det}\left(R[G]^{\times}\right) .
\end{aligned}
$$

Corollary 19 For an arbitrary finite group $G$ the quotient group

$$
\operatorname{Det}\left(S[G]^{\times}\right)^{\Delta} / \operatorname{Det}\left(R[G]^{\times}\right)
$$

has order prime to $p$.

Proof. By Theorem 16 we can find an integer $m$ which is coprime to $p$, $\mathbf{Q}_{p}$-p-elementary subgroups $H_{i}$ of $G$, integers $n_{i}$, and $\theta_{i} \in K_{0}\left(\mathbf{Q}_{p}\left[H_{i}\right]\right)$, such that

$$
m \cdot 1_{G}=\sum_{i} n_{i} \cdot \operatorname{Ind}_{H_{i}}^{G}\left(\theta_{i}\right) .
$$

Thus, given $\operatorname{Det}(x) \in \operatorname{Det}\left(S[G]^{\times}\right)^{\Delta}$, by the Frobenius module structure of $\operatorname{Det}\left(S[G]^{\times}\right)$over $K_{0}\left(\mathbf{Q}_{p}[G]\right)($ see $(17))$

$$
\operatorname{Det}(x)^{m}=\prod_{i} \operatorname{Ind}_{H_{i}}^{G}\left(\theta_{i} \cdot \operatorname{Res}_{G}^{H_{i}}(\operatorname{Det}(x))\right)^{n_{i}} .
$$

However, by the Theorem 18, we know that

$$
\theta_{i} \cdot \operatorname{Res}_{G}^{H_{i}}(\operatorname{Det}(x)) \in \operatorname{Det}\left(S\left[H_{i}\right]^{\times}\right)^{\Delta}=\operatorname{Det}\left(R\left[H_{i}\right]^{\times}\right) .
$$

Hence $\operatorname{Det}(x)^{m} \in \operatorname{Det}\left(R[G]^{\times}\right)$. 
In order to prove Theorem 13 in full generality one now has to consider the $\mathbf{Q}_{p}$-l-elementary subgroups of $G$ for the primes $l \neq p$ and then use Brauer induction to show that for each such prime $l$ the quotient $\operatorname{Det}\left(S[G]^{\times}\right)^{\Delta} / \operatorname{Det}\left(R[G]^{\times}\right)$ has order prime to $l$. In fact the argument for the primes different from $p$ is much more straightforward; the reader is referred to 6.b in [CPT1] for the details.

\section{Applications of the group logarithm}

The group logarithm has numerous applications in both algebra and arithmetic. Although here we concentrate principally on the arithmetic, we begin our account by describing some algebraic applications. Historically these came first, and also this will help us develop the notation that we will need for the subsequent arithmetic applications.

The intention is to give only a very brief overview of each chosen topic, but to highlight the role played by the group logarithm.

\subsection{Some algebraic applications}

We denote by $K_{0}(\mathbf{Z}[G])$ the Grothendieck group of finitely generated projective $\mathbf{Z}[G]$-modules. There is a natural notion of $\mathbf{Z}[G]$-rank which induces a surjective homomorphism rk : $K_{0}(\mathbf{Z}[G]) \rightarrow \mathbf{Z}$; the kernel of the rank map is called the class group of the group ring $\mathbf{Z}[G]$ and is denoted $C l(\mathbf{Z}[G])$.

In [F2] Fröhlich gave a description of $C l(\mathbf{Z}[G])$ in terms of adelic $K_{1}$-groups and described a natural isomorphism

$$
C l(\mathbf{Z}[G]) \cong \frac{\prod^{\prime} K_{1}\left(\mathbf{Q}_{p}[G]\right)}{K_{1}(\mathbf{Q}[G])^{b} \prod K_{1}\left(\mathbf{Z}_{p}[G]\right)^{b}} ;
$$

here $K_{1}(\mathbf{Q}[G])^{b}$ denotes the image of $K_{1}(\mathbf{Q}[G])$ in $\prod K_{1}\left(\mathbf{Q}_{p}[G]\right)$ (product over all primes $p) ; \prod K_{1}\left(\mathbf{Z}_{p}[G]\right)^{b}$ denotes the image of $\prod K_{1}\left(\mathbf{Z}_{p}[G]\right)$ in $\prod K_{1}\left(\mathbf{Q}_{p}[G]\right)$; and $\prod^{\prime} K_{1}\left(\mathbf{Q}_{p}[G]\right)$ denotes the restricted product, which is the subgroup of elements in $\prod K_{1}\left(\mathbf{Q}_{p}[G]\right)$ with almost all but a finite number of the entries lying in $K_{1}\left(\mathbf{Z}_{p}[G]\right)^{b}$.

Let $\mathfrak{M}$ denote a maximal $\mathbf{Z}$-order in $\mathbf{Q}[G]$ which contains $\mathbf{Z}[G]$; then $C l(\mathfrak{M})$ may be defined similarly and extension of scalars yields a surjective homomorphism $C l(\mathbf{Z}[G]) \rightarrow C l(\mathfrak{M})$; the kernel is called the kernel group and is denoted $D(\mathbf{Z}[G])$; it is independent of the particular choice of the maximal order $\mathfrak{M}$. Let $\mathfrak{M}_{p}=\mathfrak{M} \otimes \mathbf{z} \mathbf{Z}_{p}$. The kernel group can also be written in terms of $K_{1}$-groups:

$$
D(\mathbf{Z}[G]) \cong \frac{\prod K_{1}\left(\mathfrak{M}_{p}\right)^{b}}{K_{1}(\mathbf{Q}[G])^{b} \prod K_{1}\left(\mathbf{Z}_{p}[G]\right)^{b}} .
$$


However, it is often more practical to use the identification, deriving from the isomorphism Det : $K_{1}\left(\mathbf{Q}_{p}[G]\right) \rightarrow \prod_{\chi} \mathbf{Q}_{p}(\chi)^{\times}$(where the product extends over the irreducible $\mathbf{Q}_{p}^{c}$-characters of $G$ ). We let $\mathbf{Q}^{c}$ denote a chosen algebraic closure of $\mathbf{Q}$ and set $\Omega=\operatorname{Gal}\left(\mathbf{Q}^{c} / \mathbf{Q}\right)$; we let $\mathbf{Q}_{p}^{c}$ denote a chosen algebraic closure of $\mathbf{Q}_{p}$ and set $\Omega_{p}=\operatorname{Gal}\left(\mathbf{Q}_{p}^{c} / \mathbf{Q}_{p}\right)$; and once and for all we fix an embedding $\mathbf{Q}^{c} \rightarrow \mathbf{Q}_{p}^{c}$. We let $R_{G}$ resp. $R_{G, p}$ denote the ring of virtual $\mathbf{Q}^{c}$-characters resp. of virtual $\mathbf{Q}_{p}^{c}$-characters of $G$. The embedding $\mathbf{Q}^{c} \hookrightarrow \mathbf{Q}_{p}^{c}$ yields an isomorphism $R_{G} \cong R_{G, p}$ and we have natural identifications (using the Hasse-Schilling norm theorem)

$$
\begin{aligned}
K_{1}\left(\mathbf{Z}_{p}[G]\right)^{b} & =\operatorname{Det}\left(\mathbf{Z}_{p}[G]^{\times}\right), \quad K_{1}(\mathfrak{M})^{b}=\operatorname{Det}\left(\mathfrak{M}^{\times}\right)=\operatorname{Hom}_{\Omega}^{+}\left(R_{G}, O_{\mathbf{Q}^{c}}^{\times}\right), \\
K_{1}\left(\mathfrak{M}_{p}\right)^{b} & =\operatorname{Det}\left(\mathfrak{M}_{p}^{\times}\right)=\operatorname{Hom}_{\Omega_{p}}\left(R_{G}, O_{\mathbf{Q}_{p}^{c}}^{\times} ;\right.
\end{aligned}
$$

here $\operatorname{Hom}_{\Omega}^{+}\left(R_{G}, O_{\mathbf{Q}^{c}}^{\times}\right)$denotes the subgroup of $\operatorname{Hom}_{\Omega}\left(R_{G}, O_{\mathbf{Q}^{c}}^{\times}\right)$of homomorphisms whose values on the symplectic characters of $G$ are totally positive, in the sense that they are real and positive at each Archimedean place of $\mathbf{Q}^{c}$. In particular, if $G$ is a $p$-group, then for $l \neq p$, we know that $\mathbf{Z}_{l}[G]$ is a maximal $\mathbf{Z}_{l}$-order and so $\operatorname{Det}\left(\mathfrak{M}_{l}^{\times}\right)=\operatorname{Det}\left(\mathbf{Z}_{l}[G]^{\times}\right)$; and hence in this case we have the very useful and practical isomorphism:

$$
D(\mathbf{Z}[G]) \cong \frac{\operatorname{Hom}_{\Omega_{p}}\left(R_{G, p}, O_{\mathbf{Q}_{p}^{c}}^{\times}\right)}{\operatorname{Hom}_{\Omega}^{+}\left(R_{G}, O_{\mathbf{Q}^{c}}^{\times}\right) \operatorname{Det}\left(\mathbf{Z}_{p}[G]^{\times}\right)} .
$$

In particular note that this isomorphism shows how, for calculations, we can represent a class by a character function.

The exact sequence resulting from extension of scalars

$$
1 \rightarrow D(\mathbf{Z}[G]) \rightarrow C l(\mathbf{Z}[G]) \rightarrow C l(\mathfrak{M}) \rightarrow 1
$$

is crucial in understanding the class group $C l(\mathbf{Z}[G])$. On the one hand the image $C l(\mathfrak{M})$ can readily be described as a product of various kinds of class groups of rings of integers (see Theorem 3.7 in [F1] for details); and, on the other hand, the group logarithm is an excellent tool in the study of the kernel group $D(\mathbf{Z}[G])$. To illustrate this latter point we now suppose that $G$ is a $p$-group. Early on in the study of such kernel groups Ullom showed (see for instance 50.19 in [CR2] or Theorem 3.1 in $[\mathrm{U}])$ :

Theorem 20 If $G$ is a non-trivial p-group, then $D(\mathbf{Z}[G])$ is a finite p-group whose exponent divides $|G| p^{-1}$ if $p>2$ and whose exponent divides $|G| / 4$ if $p=2$.

This leads naturally to the question of when this bound for the exponent of $D(\mathbf{Z}[G])$ is attained. To begin to answer this question we introduce the notion of Swan modules: 
Definition (See [U].) Let $m$ be an integer which is coprime to $p$. The Swan module $(m, \Sigma)$ is the left (and in fact two-sided) $\mathbf{Z}[G]$-ideal generated by $m$ and the trace element $\Sigma=\sum_{g \in G} g$. Then $(m, \Sigma)$ is a projective $\mathbf{Z}[G]$-module whose class in $C l(\mathbf{Z}[G])$ lies in $D(\mathbf{Z}[G])$. These classes form a subgroup of $D(\mathbf{Z}[G])$ which is denoted $T(\mathbf{Z}[G])$ and is called the Swan subgroup of $D(\mathbf{Z}[G])$.

The class of $(m, \Sigma)$ is denoted by $[m, \Sigma]$. Suppose again that $G$ is a $p$ group; then this class is represented under the isomorphism (22) by the character function $f_{m}$ :

$$
f_{m}(\chi)=m^{(\chi, \varepsilon)}
$$

where $\varepsilon$ denotes the trivial character of $G$ and and $(\chi, \varepsilon)$ denotes the standard character inner product of $\chi$ with $\varepsilon$ (see 3.1 in [T1]).

Definition. Recall that for $n \geq 4$ the semi-dihedral group of order $2^{n}$ has presentation

$$
\left\langle\sigma, \tau \mid \sigma^{2^{n-1}}=1=\tau^{2}, \tau \sigma \tau=\sigma^{-1+2^{n-2}}\right\rangle .
$$

A 2-group is called exceptional if it is dihedral, quaternion or semi-dihedral.

Theorem 21 (Kulakoff, Alperin, Feit, Thompson) If $G$ is a p-group, then the cardinality of the set $\left\{g \in G \mid g^{p}=1\right\}$ is divisible by $p^{2}$ unless $G$ is cyclic or $p=2$ and $G$ is exceptional.

S. Ullom conjectured the following result (see [U]) which was proved by the third named author in [T2].

Theorem 22 If $p>2$ and $G$ is not cyclic, then $T(\mathbf{Z}[G])$ is a cyclic group of order $|G| p^{-1}$. If $p=2$ and $G$ is neither cyclic nor exceptional, then $T(\mathbf{Z}[G])$ is a cyclic group of order $|G| / 4$.

Remark. If $G$ is cyclic, then by Corollary 1.3 in Ch. 3 of [T1] we know that $T(\mathbf{Z}[G])=\{1\}$. If $G$ is an exceptional 2-group, then from Theorems 2.5, 2.6 loc.cit. we know that $T(\mathbf{Z}[G])=D(\mathbf{Z}[G])$ and has order two if $G$ is semidihedral or quaternion, and, if $G$ is dihedral, then $T(\mathbf{Z}[G])=D(\mathbf{Z}[G])=\{1\}$.

Sketch proof. Let $\rho$ denote the regular character of $G$. We define a map

$$
h: \operatorname{Hom}_{\Omega_{p}}\left(R_{G, p}, O_{\mathbf{Q}_{p}^{c}}^{\times}\right) \rightarrow p \mathbf{Z}_{p} \bmod p|G| \mathbf{Z}_{p}
$$

by the rule that for $\phi \in \operatorname{Hom}_{\Omega_{p}}\left(R_{G, p}, O_{\mathbf{Q}_{p}^{c}}^{\times}\right)$,

$$
h(\phi)=\log \phi\left(p \rho-\psi^{p} \rho\right) \bmod p|G| \mathbf{Z}_{p} .
$$


We note that, because $p \rho-\psi^{p} \rho$ is a rational valued character, for $\phi$ in the group $\operatorname{Hom}_{\Omega}\left(R_{G}, O_{\mathbf{Q}^{c}}^{\times}\right)$, it follows that $\phi\left(p \rho-\psi^{p} \rho\right)= \pm 1$ and so $\log \phi\left(p \rho-\psi^{p} \rho\right)=0$. For $\phi=\operatorname{Det}(z) \in \operatorname{Det}\left(\mathbf{Z}_{p}[G]^{\times}\right)$by Lemma 4 ,

$$
\begin{aligned}
h(\phi) & =\log \phi\left(p \rho-\psi^{p} \rho\right) \bmod p|G| \mathbf{Z}_{p} \\
& =\rho(p L(\operatorname{Det}(z))) \bmod p|G| \mathbf{Z}_{p}
\end{aligned}
$$

and by Theorem 3, $\rho(p L(\operatorname{Det}(z))) \in \rho\left(p \mathbf{Z}_{p}[\operatorname{Conj}[G]]\right) \subset p|G| \mathbf{Z}_{p}$. It therefore follows that $h$ induces a map (denoted by the same symbol)

$$
h: D(\mathbf{Z}[G]) \rightarrow p \mathbf{Z}_{p} \bmod p|G| \mathbf{Z}_{p}
$$

We now evaluate $h$ on the class $[1+p, \Sigma]$ by evaluating $h\left(f_{1+p}\right)$ :

$$
\begin{aligned}
h\left(f_{1+p}\right) & =\log \left(f_{1+p}\left(p \rho-\psi^{p} \rho\right)\right) \bmod |G| \mathbf{Z}_{p} \\
& =\log \left((1+p)^{\left(p \rho-\psi^{p} \rho, \varepsilon\right)}\right) \bmod |G| \mathbf{Z}_{p}
\end{aligned}
$$

and the character inner product $\left(p \rho-\psi^{p} \rho, \varepsilon\right)=(p \rho, \varepsilon)-\left(\psi^{p} \rho, \varepsilon\right)$ can be evaluated by

$$
\begin{aligned}
(p \rho, \varepsilon) & =\frac{1}{|G|} \sum_{g} p \rho(g) \varepsilon\left(g^{-1}\right)=p \\
\left(\psi^{p} \rho, \varepsilon\right) & =\frac{1}{|G|} \sum_{g} \psi^{p} \rho(g) \varepsilon\left(g^{-1}\right)=\frac{1}{|G|} \sum_{g} \rho\left(g^{p}\right)=\sum_{g \mid g^{p}=1} 1 .
\end{aligned}
$$

The result then follows at once from the Kulakoff-Alperin-Feit-Thompson theorem.

The involution $g \longmapsto g^{-1}$ of the group $G$ induces involutions denoted $c \mapsto \bar{c}$ on both $C l(\mathbf{Z}[G])$ and $D(\mathbf{Z}[G])$. We define

$$
\begin{aligned}
D(\mathbf{Z}[G])^{+} & =\{d \in D(\mathbf{Z}[G]) \mid \bar{d}=d\} \\
D(\mathbf{Z}[G])^{-} & =\{d \in D(\mathbf{Z}[G]) \mid \bar{d}=-d\} .
\end{aligned}
$$

For the sake of simplicity we shall suppose for the remainder of this subsection that $p>2$. We then have the direct decomposition

$$
D(\mathbf{Z}[G])=D(\mathbf{Z}[G])^{+} \oplus D(\mathbf{Z}[G])^{-} .
$$

In [O6] Oliver constructs a map

$$
\Delta: D(\mathbf{Z}[G])^{+} \rightarrow K_{0}(\mathbf{Q}[G]) T(\mathbf{Z}[G])
$$


where $K_{0}(\mathbf{Q}[G])$ acts on classes as in 5.1. Let $A(\mathbf{Q}[G])$ denote the Artin ideal of $K_{0}(\mathbf{Q}[G])$ obtained by inducing up the rational characters $K_{0}(\mathbf{Q}[C])$ of cyclic subgroups $C$ of $G$. Oliver then shows further that

$$
K_{0}(\mathbf{Q}[G]) T(\mathbf{Z}[G]) \cong \frac{K_{0}(\mathbf{Q}[G])}{A(\mathbf{Q}[G])}
$$

and that if furthermore $p$ is a regular prime number, then in fact $\Delta$ is an isomorphism (see Theorem 3.4 in [O6]). This then underlines the central role played by Swan modules in the study of class groups of group rings.

To conclude this subsection we note that in Theorem 3.8 of [O6] Oliver gives a closed formula for the cardinality of the group $D(\mathbf{Z}[G])^{-}$.

\subsection{The Fröhlich conjecture}

For a number field $K$ we let $O_{K}$ denote the ring of algebraic integers of $K$. We consider a finite Galois extension $N / K$ of number fields and write $G=$ $\operatorname{Gal}(N / K)$. The group ring $\mathbf{Z}[G]$ acts on $O_{K}$ in the natural way, and by Noether's theorem $O_{N}$ is a projective $\mathbf{Z}[G]$-module if, and only if, $N / K$ is at most tamely ramified. We suppose henceforth that the extension $N / K$ is tame, and so we may consider the virtual class

$$
\left[O_{N}\right]-\left[O_{K}[G]\right] \in C l(\mathbf{Z}[G])
$$

From the previous subsection we recall the K-theoretic description

$$
C l(\mathbf{Z}[G])=\frac{\prod^{\prime} K_{1}\left(\mathbf{Q}_{l}[G]\right)}{K_{1}(\mathbf{Q}[G])^{b} \prod \operatorname{Det}\left(\mathbf{Z}_{l}[G]^{\times}\right)}
$$

and for each prime $l$ we have

$$
K_{1}\left(\mathbf{Q}_{l}[G]\right)=\operatorname{Det}\left(\mathbf{Q}_{l}[G]^{\times}\right)=\prod_{\chi} \mathbf{Q}_{l}(\chi)^{\times} .
$$

Let $\Lambda(s, \chi)$ denote the Artin L-function associated to the character $\chi$ of $G$ (see $[\mathrm{M}]$ for details); then $\Lambda(s, \chi)$ satisfies a functional equation

$$
\Lambda(s, \chi)=W(\chi) \Lambda(1-s, \bar{\chi})
$$

where the constant $W(\chi)$, which is called the Artin root number, lies on the unit circle in the complex plane. Note that if $\chi$ is a real valued character of $G$, then $W(\chi)^{2}=1$ and so $W(\chi)= \pm 1$. We then define the class $\left[W_{N / K}\right] \in C l(\mathbf{Z}[G])$ to be the class, represented under the isomorphism (25) and the description $K_{1}\left(\mathbf{Q}_{p}[G]\right)$ in (26), by the element

$$
\prod_{l} \prod_{\chi} w_{\chi, l} \in \prod^{\prime} K_{1}\left(\mathbf{Q}_{l}[G]\right)
$$


where for irreducible $\mathbf{Q}^{c}$-characters $\chi$ of $G$

$$
w_{\chi, l}= \begin{cases}W(\chi) \text { if } \chi \text { is symplectic and } p|| G \mid \\ 1 & \text { otherwise. }\end{cases}
$$

Based on a considerable number of known examples, Fröhlich conjectured the following result, which was proved by the third author (see [T4]) :

Theorem 23 There is an equality in $C l(\mathbf{Z}[G])$

$$
\left[O_{N}\right]-\left[O_{K}[G]\right]=\left[W_{N / K}\right] .
$$

We now very briefly indicate the role of the group logarithm in the proof of this result. For illustrative purposes we henceforth suppose $G$ to be a $p$-group.

In Theorem 6 of [F1] Fröhlich gave an explicit representative

$$
\prod \kappa_{l} \in \prod^{\prime} K_{1}\left(\mathbf{Q}_{l}[G]\right)
$$

for the class $\left[O_{N}\right]-\left[O_{K}[G]\right]-\left[W_{N / K}\right]$ with $\kappa_{l}=1$ if $l \neq p$.

Step 1. A key-ingredient in the definition of the $\kappa_{l}$ is the Galois Gauss sum $\tau_{N / K}$ which is related to the Artin root number $W(\chi)$ by the formula (see page 126 in $[\mathrm{F} 1])$

$$
W_{\infty}(\chi) W(\chi) N \mathfrak{f}(\chi)^{1 / 2}=\tau_{N / K}(\chi) .
$$

As in the corollary to Theorem 18 in [F1], we know that the $\tau_{N / K}(\chi)$ can be written naturally as a product

$$
\tau_{N / K}(\chi)=\prod_{l} \tau_{l}(\chi)
$$

and almost all $\tau_{l}(\chi)=1$.

Recall that we have fixed an embedding $\mathbf{Q}^{c} \rightarrow \mathbf{Q}_{p}^{c}$. For $x \in \mathbf{Q}^{c}$ we write $(x)_{p}$ for the image of $x$ in $\mathbf{Q}_{p}^{c}$. By using Frohlich's Galois action formula for Gauss sums (see Theorem 20B in [F1]), it is relatively straightforward to idenitfy where for $l \neq p$ the element $\left(\tau_{l}\right)_{p}$ lies, and we get

$$
\prod_{\chi}\left(\tau_{l}(\chi)\right)_{p} \in \prod_{\chi} \mathbf{Q}_{p}\left(\zeta_{l}\right)(\chi)^{\times}=K_{1}\left(\mathbf{Q}_{p}\left(\zeta_{l}\right)[G]\right)=\operatorname{Det}\left(\mathbf{Q}_{p}\left(\zeta_{l}\right)[G]^{\times}\right) .
$$

For those $\tau_{l} \neq 1$, it is convenient to make an adjustment and work with the modified local Gauss sums $\tau_{l}^{*}=\xi_{l} \tau_{l}$ for a root of unity valued character function $\xi_{l} \in \operatorname{Hom}_{\Omega}^{+}\left(R_{G}, \mu\right)$ where the precise formula for $\xi_{l}$ is given on page151 of [F1]. (This change is very similar to the change from Deligne's $\varepsilon$-constants to his 
$\varepsilon_{0}$-constants in $[\mathrm{De}]$.) Moreover, if $\mathfrak{M}_{p, G}$ denotes a maximal $\mathbf{Z}_{p}\left[\zeta_{l}\right]$-order in $\mathbf{Q}_{p}\left(\zeta_{l}\right)[G]$, then, since $l \neq p$, the $\tau_{l}^{*}(\chi)$ are all $p$-units, and it follows that

$$
\left(\tau_{l}^{*}\right)_{p} \in K_{1}\left(\mathfrak{M}_{p, G}\right)^{b}=\operatorname{Det}\left(\mathfrak{M}_{p, G}^{\times}\right) .
$$

The key-point in the proof of Theorem 23 is that we can show that $\left(\tau_{l}^{*}\right)_{p}$ lies in the subgroup $\operatorname{Det}\left(\mathbf{Z}_{p}\left[\zeta_{l}\right][G]^{\times}\right)$and for this we make crucial use the group $\operatorname{logarithm} L$. (Note that this result would not be true for the unmodified Galois Gauss sum.) Since $\operatorname{Det}\left(\mathbf{Z}_{p}\left[\zeta_{l}\right][G]^{\times}\right)$has finite index in $\operatorname{Det}\left(\mathfrak{M}_{p, G}^{\times}\right)$and since the the group logarithm take values in the uniquely divisible group $\mathbf{Q}_{p}\left(\zeta_{l}\right)[\operatorname{Conj}(G)]$, $\nu$ extends naturally to a map

$$
\widetilde{\nu}: \operatorname{Det}\left(\mathfrak{M}_{p, G}^{\times}\right) \rightarrow \mathbf{Q}_{p}\left(\zeta_{l}\right)[\operatorname{Conj}(G)] .
$$

We wish to show that $\widetilde{\nu}\left(\left(\tau_{l}^{*}\right)_{p}\right)$ lies in the integral lattice $\mathbf{Z}_{p}\left[\zeta_{l}\right][\operatorname{Conj}(G)]$. To see this we write

$$
\widetilde{\nu}\left(\left(\tau_{p}^{*}\right)_{l}\right)=\sum_{c(g) \in \operatorname{Conj}(G)} t_{c(g)} c(g) \in \mathbf{Q}_{p}\left(\zeta_{l}\right)[\operatorname{Conj}(G)] .
$$

Then, by Fourier inversion, we may calculate $t_{c(g)}$ :

$$
t_{c(g)}=\frac{1}{|c(g)|} \sum_{\chi} \chi\left(\widetilde{\nu}\left(\tau_{p}\right)_{l}\right) \chi\left(g^{-1}\right)
$$

summing over the irreducible characters of $G$. By the explicit formula (9) in Lemma 4 this may be written as

$$
t_{c(g)}=\frac{1}{|c(g)|} \sum_{\chi}\left[\log \left(\tau_{p}\right)_{l}(\chi)-\frac{1}{p} \log \left(\tau_{p}^{F}\right)_{l}\left(\psi^{p} \chi\right)\right] \chi\left(h^{-1}\right)
$$

where $F$ denotes the Frobenius of $\mathbf{Q}_{p}\left(\zeta_{l}\right) / \mathbf{Q}_{p}$. Using again the Galois action formula for Gauss sums loc. cit. we see that $\tau_{p}^{F}(\chi)$ differs from $\tau_{p}(\chi)$ by a root of unity. So by the above this expression can be written as

$$
t_{c(g)}=\frac{1}{p|c(g)|} \sum_{\chi}\left[\log \left(\tau_{p}\right)_{l}\left(p \chi-\psi^{p} \chi\right)\right] \chi\left(g^{-1}\right) .
$$

This sum can then be readily evaluated using the Hasse-Davenport Gauss sum formulas, and we find that each $t_{c(g)} \in \mathbf{Z}_{p}\left[\zeta_{l}\right]$, as required. See Ch. IV Sect.5 in [F1] for details.

Step 2. The above work essentially shows that we can find $z_{l} \in \mathbf{Z}_{p}[G]^{\times}$ so that $\operatorname{Det}(z) \tau_{l}^{*} \in \operatorname{ker} \widetilde{\nu}$. As $G$ is a $p$-group, $\mathbf{Z}_{p}[G]^{\times}=\mu_{p-1} \times\left(1+J_{G}\right)$ and we extend $\nu$ from $\operatorname{Det}\left(1+J_{G}\right)$ to $\nu^{\prime}$ on $\operatorname{Det}\left(\mathbf{Z}_{p}[G]^{\times}\right)$by setting $\nu^{\prime}$ to be 0 on $\operatorname{Det}\left(\mu_{p-1}\right)$. Then, with this convention, by Theorem 5 we now get that 
$\operatorname{ker} \nu^{\prime}=\mu_{p-1} \times \operatorname{Det}(G)$; however, by the reasoning after in Lemma 4, we see that ker $\widetilde{\nu}$ is the much larger group $\operatorname{Det}\left(\mathfrak{M}_{p, G}^{\times}\right)_{\text {tor }}$ consisting of all the elements in $\operatorname{Det}\left(\mathfrak{M}_{p, G}^{\times}\right)$of finite order. The next step in the proof consists of finding virtual characters $\chi_{i}$ of $G$ and ideals $\mathfrak{a}_{i}$ of $\mathbf{Z}_{p}\left[\chi_{i}\right]$ with the property that the following sequence is exact:

$$
1 \rightarrow \operatorname{ker} \nu \rightarrow \operatorname{ker} \widetilde{\nu} \rightarrow \prod_{i} \mathbf{z}_{p}\left[\chi_{i}\right]^{\times} \bmod \mathfrak{a}_{i}
$$

where the right-hand arrow is induced by the product of evaluation of determinants on $\chi_{i}$ followed by reduction $\bmod \mathfrak{a}_{i}$. We then conclude the proof by showing that for each $i$

$$
\operatorname{Det}\left(z_{l}\right)\left(\chi_{i}\right) \tau_{l}^{*}\left(\chi_{i}\right) \equiv 1 \bmod \mathfrak{a}_{i} .
$$

This then allows us to deduce that $\tau_{l}^{*} \in \operatorname{Det}\left(\mathbf{Z}_{p}[G]^{\times}\right)$, as required.

Step 3. Fröhlich's description of the representative $\kappa_{p}$ allows us to write it as

$$
\kappa_{p}=\lambda_{p} \prod_{p \neq l}\left(\tau_{l}^{*}(\chi)\right)_{p}
$$

Here the term $\lambda_{p}$ is made up of the corestriction of non-abelian resolvents and the Gauss sum $\tau_{p}(\chi)$. Using some elementary algebra and the Galois action formula for Gauss sums and resolvents it is relatively straightforward to show that $\lambda_{p} \in \operatorname{Det}\left(O_{T}[G]^{\times}\right)$for some tame extension $T$ of $\mathbf{Q}_{p}$; and the work in Steps 1 and 2 shows that

$$
\prod_{p \neq l}\left(\tau_{l}^{*}(\chi)\right)_{p} \in \operatorname{Det}\left(O_{N}[G]^{\times}\right)
$$

for some non-ramified extension $N$ of $\mathbf{Q}_{l}$. Increasing $T$ if necessary to a larger tame and Galois extension of $\mathbf{Q}_{l}$, we have shown that

$$
\kappa_{p} \in \operatorname{Det}\left(O_{T}[G]^{\times}\right) \cap K_{1}\left(\mathbf{Q}_{p}[G]\right) .
$$

If we set $\Delta=\operatorname{Gal}\left(T / \mathbf{Q}_{p}\right)$, then this implies that $\kappa_{p} \in \operatorname{Det}\left(O_{T}[G]^{\times}\right)^{\Delta}$ and, by an easy extension of the fixed point result Theorem 18 (see for instance Ch 8 in [T1]) we then have

$$
\kappa_{p} \in \operatorname{Det}\left(O_{T}[G]^{\times}\right)^{\Delta}=\operatorname{Det}\left(O_{T}^{\Delta}[G]^{\times}\right)=\operatorname{Det}\left(\mathbf{Z}_{p}[G]^{\times}\right)
$$

and this then shows that the representative $\kappa_{p}$ lies in the denominator of the description of $C l(\mathbf{Z}[G])$ given in (25).

In summary, note that the group logarithm has played two essentially different roles in the proof of the Fröhlich Conjecture: firstly, in showing that the adjusted Gauss sum is a group ring determinant; and secondly through the use the fixed point theorem for determinants. 


\subsection{Equivariant second Chern classes and Riemann-Roch}

Next we give a flavour of some new applications of the above results on Det and $S K_{1}$ for the calculation of Euler characteristics of equivariant sheaves on arithmetic surfaces.

Consider a finite group $G$, an irreducible, regular two dimensional regular scheme $Y$ with structure map $f: Y \rightarrow \operatorname{Spec}(\mathbf{Z})$ which is projective and flat. Suppose that all the fibers of $f$ are reduced with smooth irreducible components and that the fibers over all the divisors of $|G|$ are irreducible and smooth. Let $\mathcal{E}$ denote an $\mathcal{O}_{Y}[G]$-vector bundle, i.e a coherent sheaf of $\mathcal{O}_{Y}[G]$-modules on $Y$ which is locally free. We can then form the projective Euler characteristic of $\mathcal{E}$, denoted $\chi(Y, \mathcal{E})$, which lies in $C l(\mathbf{Z}[G])$. This class is constructed by first finding a perfect $\mathbf{Z}[G]$-complex, which is quasi-isomorphic to a Cech complex which computes the cohomology of $\mathcal{E}$. One then forms the alternating sum of the classes of the terms in the perfect complex; see [CE] for details. We now indicate briefly the role of group logarithmic techniques in forming a second Chern class, which can be used to calculate such projective Euler characteristics.

We start by recalling some constructions due to Beilinson and Parshin. For $0 \leq i \leq 2$ we let $\eta_{i}$ denote a point of $Y$ of codimension $i$. A Parshin pair is a pair of points $\left(\eta_{i}, \eta_{j}\right)$ with $i<j$ and with $\eta_{j}$ on the closure of $\eta_{i}$. A Parshin triple is a triple of points $\left(\eta_{0}, \eta_{1}, \eta_{2}\right)$ with $\eta_{2}$ on the closure of $\eta_{1}$. (Since $Y$ is irreducible there is only one generic point on $Y$ and so we do not need to impose any additional conditions). We can then form the completed ring $\widehat{\mathcal{O}}_{Y, \eta_{i}}$; following Parshin and Beilinson for a Parshin pair $\left(\eta_{i}, \eta_{j}\right)$ resp. a Parshin triple $\left(\eta_{0}, \eta_{1}, \eta_{2}\right)$ we can form the muticompletion $\widehat{\mathcal{O}}_{Y, \eta_{i} \eta_{j}}$ resp. $\widehat{\mathcal{O}}_{Y, \eta_{0} \eta_{1} \eta_{2}}$. For given $0 \leq i \leq 2$ we then form the products of (Quillen) $K_{2}$-groups

$$
\begin{aligned}
K_{2}\left(\mathbb{A}_{Y, 012}[G]\right) & =\prod K_{2}\left(\widehat{\mathcal{O}}_{Y, \eta_{0} \eta_{1} \eta_{2}}[G]\right), \\
K_{2}\left(\mathbb{A}_{Y, i j}[G]\right) & =\prod K_{2}\left(\widehat{\mathcal{O}}_{Y, \eta_{i} \eta_{j}}[G]\right) .
\end{aligned}
$$

Here the first product extends over all Parshin triples $\left(\eta_{0}, \eta_{1}, \eta_{2}\right)$ on $Y$, and the second product extends over all Parshin pairs $\left(\eta_{i}, \eta_{j}\right)$ on $Y$. For any such $i<j$ there is a natural map $\widehat{\mathcal{O}}_{Y, \eta_{i} \eta_{j}} \rightarrow \widehat{\mathcal{O}}_{Y, \eta_{0} \eta_{1} \eta_{2}}$ and so we have maps $K_{2}\left(\mathbb{A}_{Y, i j}[G]\right) \rightarrow$ $K_{2}\left(\mathbb{A}_{Y, 012}[G]\right)$ and we write $K_{2}\left(\mathbb{A}_{Y, i j}[G]\right)^{b}$ for the image of $K_{2}\left(\mathbb{A}_{Y, i j}[G]\right)$ in $K_{2}\left(\mathbb{A}_{Y, 012}[G]\right)$. Following Parshin and Beilinson, we define various restricted adelic subgroups $K_{2}^{\prime}\left(\mathbb{A}_{Y, 012}[G]\right), K_{2}^{\prime}\left(\mathbb{A}_{Y, i j}[G]\right)$ (see Definition 2.2 in [CPT3]) and we define the second equivariant Chow group

$$
\mathrm{CH}_{\mathbb{A}}^{2}(Y[G])=\frac{K_{2}^{\prime}\left(\mathbb{A}_{Y, 012}[G]\right) \cdot \prod_{0 \leq i, j \leq 2} K_{2}^{\prime}\left(\mathbb{A}_{Y, i j}[G]\right)^{b}}{\prod_{0 \leq i, j \leq 2} K_{2}^{\prime}\left(\mathbb{A}_{Y, i j}[G]\right)^{b}} .
$$


Note at this point that we can also interpret the class group $C l(\mathbf{Z}[G])$ as a first equivariant Chow group $\mathrm{CH}_{\mathbb{A}}^{1}(\operatorname{Spec}(\mathbf{Z})[G])$. We will assume for simplicity that the group ring is split, i.e that $\mathbf{Q}[G] \cong M_{n_{i}}\left(L_{i}\right)$ where $L_{i}$ are (commutative) fields. Then in Section 4 of [CPT3] we describe a push down map

$$
f_{*}: \mathrm{CH}_{\mathbb{A}}^{2}(Y[G]) \rightarrow \mathrm{CH}_{\mathbb{A}}^{1}(\operatorname{Spec}(\mathbf{Z})[G])=C l(\mathbf{Z}[G]) .
$$

For a suitable equivariant vector $\mathcal{O}_{Y}[G]$-bundle $\mathcal{E}$ of rank $n$ we now sketch the construction of a second Chern class $c_{2}(\mathcal{E}) \in \mathrm{CH}_{\mathbb{A}}^{2}(Y[G])$.

Definition. For each point $\eta_{i}$ on $Y$ we choose a basis $\left\{e_{i}\right\}$ of $\mathcal{E} \otimes_{\mathcal{O}_{Y}} \widehat{\mathcal{O}}_{Y, \eta_{i}}$ over $\widehat{\mathcal{O}}_{Y, \eta_{i}}[G]$; we then have transition matrices $\lambda_{i j} \in G L_{n}\left(\widehat{\mathcal{O}}_{Y, \eta_{i} \eta_{j}}[G]\right)$. We say that $\mathcal{E}$ has elementary structure if the bases can be chosen so that all the $\lambda_{i j}$ considered in $G L\left(\widehat{\mathcal{O}}_{Y, \eta_{i} \eta_{j}}[G]\right)$ actually belong to the subgroup of elementary matrices $E\left(\widehat{\mathcal{O}}_{Y, \eta_{i} \eta_{j}}[G]\right)$.

We suppose henceforth that $\mathcal{E}$ has elementary structure.

The importance of elementary structure is that it allows us to form a second Chern class as follows: for each triple $0 \leq i, j \leq 2$ we have the corresponding Steinberg sequence (see $[\mathrm{R}]$ ) associated to the ring $\widehat{\mathcal{O}}_{Y, \eta_{i} \eta_{j}}[G]$ :

$$
1 \rightarrow K_{2}\left(\widehat{\mathcal{O}}_{Y, \eta_{i} \eta_{j}}[G]\right) \rightarrow \operatorname{St}\left(\widehat{\mathcal{\mathcal { O }}}_{Y, \eta_{i} \eta_{j}}[G]\right) \rightarrow E\left(\widehat{\mathcal{O}}_{Y, \eta_{i} \eta_{j}}[G]\right) \rightarrow 1
$$

We can now choose lifts $\widetilde{\lambda}_{i j}$ of $\lambda_{i j}$ in $S t\left(\widehat{\mathcal{O}}_{Y, \eta_{i} \eta_{j}}[G]\right)$ and we define the "2-cocycle"

$$
z\left(\eta_{0}, \eta_{1}, \eta_{2}\right)=\widetilde{\lambda}_{02} \cdot\left(\widetilde{\lambda}_{12}\right)^{-1} \cdot\left(\widetilde{\lambda}_{01}\right)^{-1} \in K_{2}\left(\widehat{\mathcal{O}}_{Y, \eta_{0} \eta_{1} \eta_{2}}[G]\right) .
$$

In fact one can show that these lifts can be chosen so that $\prod z\left(\eta_{0}, \eta_{1}, \eta_{2}\right)$ lies in the restricted adelic product $K_{2}^{\prime}\left(\mathbb{A}_{Y, 012}[G]\right)$. The second equivariant Chern class $c_{2}(\mathcal{E}) \in \mathrm{CH}_{\AA}^{2}(Y[G])$ is then defined to be the class represented in (27). Here of course, among other things, one needs to check that the class is independent of the bases $\left\{e_{i}\right\}$ and of the lifts of the corresponding transition matrices used. The main result of [CPT3] then is:

Theorem 24 (Non-commutative adelic Riemann-Roch) Under the above assumptions, there is an equality in $C l(\mathbf{Z}[G])$

$$
\chi(Y, \mathcal{E})=-f_{*}\left(c_{2}(\mathcal{E})\right) .
$$

(Note here that the assumption that $\mathcal{E}$ has an elementary structure implies that the appropriate generalization of the first Chern class of $\mathcal{E}$ is zero; this then explains the shape of the identity above. Indeed, this agrees with the shape of the classical Riemann-Roch formula for vector bundles of virtual rank zero and trivial determinant.) 
The group logarithm is vital in this work for dealing with elementary structures. Indeed, the proof of the Riemann-Roch theorem in [CPT3] proceeds by an elaborate reduction to the case of the projective line $Y=\mathbf{P}_{\mathbf{Z}}^{1}$. There we need to be able to show that certain $\mathcal{O}_{\mathbf{P}_{\mathbf{Z}}^{1}}[G]$-bundles on $\mathbf{P}_{\mathbf{Z}}^{1}$ which are constructed by gluing have an elementary structure. Now notice that given transition matrices $\left\{\lambda_{i j}\right\}$ as above, the group logarithm work on determinants can help determine when the $\operatorname{Det}\left(\lambda_{i j}\right)=1$; this then gives $\lambda_{i j} \in S L_{n}\left(\widehat{\mathcal{O}}_{Y, \eta_{i} \eta_{j}}[G]\right)$, where $S L_{n}\left(\widehat{\mathcal{O}}_{Y, \eta_{i} \eta_{j}}[G]\right)$ denotes the kernel of Det on $G L_{n}\left(\widehat{\mathcal{O}}_{Y, \eta_{i} \eta_{j}}[G]\right)$. In order to determine whether $\lambda_{i j} \in E\left(\widehat{\mathcal{O}}_{Y, \eta_{i} \eta_{j}}[G]\right)$ we need to understand the quotient group

$$
S K_{1}\left(\widehat{\mathcal{O}}_{Y, \eta_{i} \eta_{j}}[G]\right)=\frac{S L\left(\widehat{\mathcal{O}}_{Y, \eta_{i} \eta_{j}}[G]\right)}{E\left(\widehat{\mathcal{O}}_{Y, \eta_{i} \eta_{j}}[G]\right)}
$$

Here we use our work on the description of $S K_{1}(R[G])$ for more general $p$-adic rings $R$ to describe some of the groups $S K_{1}\left(\widehat{\mathcal{O}}_{Y, \eta_{i} \eta_{j}}[G]\right)$ and show that the bundles we use have elementary structure.

\section{References}

[CE] T. Chinburg and B. Erez, Equivariant Euler-Poincaré characteristics and tameness. Journées Arithmétiques, 1991 (Geneva). Astérisque No. 209 (1992), 13, 179-194.

[CPT1] T. Chinburg, G. Pappas and M. J. Taylor, $K_{1}$ of a $p$-adic group ring I: the determinantal image, J. Algebra 326 (2011), 74-112.

[CPT2] T. Chinburg, G. Pappas and M. J. Taylor, $K_{1}$ of a $p$-adic group ring I: the kernel of the determinant, to appear.

[CPT3] T. Chinburg, G. Pappas and M. J. Taylor, Adelic Riemann-Roch, to appear.

[CR1] C. Curtis, I. Reiner, Methods of representation theory. Vol. I. With applications to finite groups and orders. Reprint of the 1981 original. Wiley Classics Library. A Wiley-Interscience Publication. John Wiley \& Sons, Inc., New York, 1990. xxiv+819 pp.

[CR2] C. Curtis, I. Reiner, Methods of representation theory. Vol. II. With applications to finite groups and orders. Pure and Applied Mathematics (New York). A Wiley-Interscience Publication. John Wiley \& Sons, Inc., New York, 1987. xviii+951 pp. 
[De] P. Deligne, Les constantes des équations fonctionnelles des fonctions L. Modular functions of one variable, II (Proc. Internat. Summer School, Univ. Antwerp, Antwerp, 1972), pp. 501-597. Lecture Notes in Math., Vol. 349, Springer, Berlin, 1973.

[D] A. Dress, Induction and structure theorems for orthogonal representations of finite groups. Ann. of Math. (2) 102 (1975), no. 2, 291-325.

[F1] A. Fröhlich, Galois module structure of algebraic integers. Ergebnisse der Mathematik und ihrer Grenzgebiete (3), 1. Springer-Verlag, Berlin, 1983. $\mathrm{x}+262 \mathrm{pp}$.

[F2] A. Fröhlich, Arithmetic and Galois module structure for tame extensions. J. Reine Angew. Math. 286/287 (1976), 380-440.

[IV] D. Izychev and O. Venjakob, Galois invariants of $K_{1}$-groups of Iwasawa algebras, Preprint, arXiv:1006.5357

[Ka1] M. Kakde, $K_{1}$ of some Iwasawa algebras, Preprint, arXiv:1005.4152

[Ka2] M. Kakde, $K_{1}$ of some non-commutative group rings, Preprint, arXiv:1003.3772

[Ka3] M. Kakde, The main conjecture of Iwasawa theory for totally real fields, Preprint, arXiv:1008.0142

[K] K. Kato, $K_{1}$ of some non-commutative completed group rings. KTheory 34 (2005), no. 2, 99-140.

[L] T. Y. Lam, Induction theorems for Grothendieck groups and Whitehead groups of finite groups. Ann. Sci. École Norm. Sup. (4) 11968 91-148.

[M] J. Martinet, Character theory and Artin L-functions, in "Algebraic number fields: L-functions and Galois properties" (Proc. Sympos., Univ. Durham, Durham, 1975), pp. 1-87. Academic Press, London, 1977.

[O1] R. Oliver, $S K_{1}$ for finite groups rings. I. Invent. Math. 57 (1980), no. 2, 183-204; correction ibid 64 (1981), no. 1, 167-169.

[O2] R. Oliver, $S K_{1}$ for finite group rings. II. Math. Scand. 47 (1980), no. $2,195-231$. 
[O3] R. Oliver, $S K_{1}$ for finite group rings. III. Algebraic K-theory, Evanston 1980 (Proc. Conf., Northwestern Univ., Evanston, Ill., 1980), pp. 299337, Lecture Notes in Math., 854, Springer, Berlin, 1981.

[O4] R. Oliver, $S K_{1}$ for finite group rings. IV. Proc. London Math. Soc. (3) 46 (1983), no. 1, 1-37.

[O5] R. Oliver, Whitehead groups of finite groups. London Mathematical Society Lecture Note Series, 132. Cambridge University Press, Cambridge, 1988. viii+349 pp.

[O6] R. Oliver, Projective class groups of integral group rings: a survey. Orders and their applications (Oberwolfach, 1984), 211-232, Lecture Notes in Math., 1142, Springer, Berlin, 1985.

[RW1] J. Ritter and A. Weiss, The integral logarithm in Iwasawa theory: an exercise. J. Théor. Nombres Bordeaux 22 (2010), no. 1, 197-207.

[RW2] J. Ritter and A. Weiss, On the "main conjecture" of equivariant Iwasawa theory. J. Amer. Math. Soc. 24 (2011), no. 4, 1015-1050.

[R] J. Rosenberg, Algebraic K-theory and its applications. Graduate Texts in Mathematics, 147. Springer-Verlag, New York, 1994. x+392 pp.

[S] J.-P. Serre, Représentations linéaires des groupes finis. Third revised edition. Hermann, Paris, 1978. 182 pp.

[Sn] V. Snaith, Topological methods in Galois representation theory. Canadian Mathematical Society Series of Monographs and Advanced Texts. A Wiley-Interscience Publication. John Wiley \& Sons, Inc., New York, 1989. $\mathrm{xvi}+299 \mathrm{pp}$.

[SV] P. Schneider, O. Venjakob, $K_{1}$ of certain Iwasawa algebras, after Kakde. This volume.

[T1] M. J. Taylor, Classgroups of group rings. London Mathematical Society Lecture Note Series, 91. Cambridge University Press, Cambridge, 1984. xiii+119 pp.

[T2] M. J. Taylor, Locally free classgroups of groups of prime power order. J. Algebra 50 (1978), no. 2, 463-487.

[T3] M. J. Taylor, A logarithmic approach to classgroups of integral group rings. J. Algebra 66 (1980), no. 2, 321-353. 
[T4] M. J. Taylor, On Fröhlich's conjecture for rings of integers of tame extensions. Invent. Math. 63 (1981), no. 1, 41-79.

[U] S. Ullom, Classgroups of group rings, in "Algebraic number fields: L-functions and Galois properties" (Proc. Sympos., Univ. Durham, Durham, 1975), pp. 497-524. Academic Press, London, 1977.

[W1] C. T. C. Wall, On the classification of Hermitian forms. III. Complete semilocal rings. Invent. Math. 19 (1973), 59-71.

[W2] C. T. C. Wall, Norms of units in group rings. Proc. London Math. Soc. (3) 29 (1974), 593-632.

T. Chinburg,

Dept. of Math.,

University of Pennsylvania,

Philadelphia, PA 19104, USA

ted@math.upenn.edu

G. Pappas,

Dept. of Math.,

Michigan State University,

E. Lansing, MI 48824, USA

pappas@math.msu.edu

M. J. Taylor,

Merton College,

Oxford University,

Oxford, OX1 4JE, UK

martin.taylor@merton.ox.ac.uk 